\title{
Literatur
}

1. PILz, W. und A. T. Boo, diese Z. 5., 173 (1967). - 2. Przz, W., Hoppe-Seyler's Z. physiol. Chem. 345, 80 (1966). - 3. Pilz, W. und I. Johann, Z. analyt. Chem. 215, 105 (1965). - 4. Pilz, W., Z. analyt. Chem. 193, 338 (1963). - 5. Zlatkis, A., B. ZACK und A. J. Boyle, J. Laborat. Clin. Med., S. Louis 41, 486 (1953). 6. PiLz, W., Z. analyt. Chem., im Druck. - 7. PILz, W. und H. HörLEIN, Hoppe-Seyler's Z. physiol. Chem. 330, 212 (1963). 8. Hörlein, H. und W. Prlz, Hoppe-Seyler's Z. physiol. Chem. 327, 256 (1962); PILZ, W. und H. HöRleIN, HoppeSeyler's Z. physiol. Chem. 330, 212 (1963). - 9. Pruz, W., Hoppe-
Seylers' Z. physiol. Chem. 328, 1 (1962); Przz, W., Mikrochim. Acta (Wien), 614 (1961). - 10. Przz, W., Zschr. exper. Med. 132, 310 (1959). - 11. Prlz, W. und I. JohanN, diese Z. 4, 215 (1966). - 12. Kalow, W. und K. Genest, Canad. J. Biochem. Physiol. 35, 339 (1957). - 13. Przz, W., I. Johann und E. StelzL, Klin. Wschr., 43, 1227 (1965). - 14. Przz, W., Hoppe-Seyler's Z. physiol. Chem. 335, 221 (1964). - 15. PrLz, W., H. HörLeIN und E. Stelzh, Hoppe-Seyler's Z. physiol. Chem., 345, 65 (1956). 16. Priz, W., in Vorbereitung.

\section{Ein neuer Redoxkatalysator für die Blutzuckerbestimmung mit Glucoseoxydase}

\author{
Von A. Härtel, K. Fabel-Schutte, H. Lang und W. Rick \\ Aus der Biocbemischen Abteilung der E. Merck AG Darmstadt und der 1. Mediqinischen Universitätsklinik Düsseldorf \\ (Direktor: Prof. Dr. F. Grosse-Brockboff)
}

(Eingegangen am 25. April 1967)

\begin{abstract}
Für die Blutzuckerbestimmung mit Glucoseoxydase kann an Stelle der bisher gebräuchlichen Peroxydase ein Gemisch von Jodid und Vanadat verwendet werden. In bezug auf Farbausbeute, Reaktionsgeschwindigkeit und Störanfälligkeit ist dieser anorganische Katalysator der Peroxydase gleichwertig, er übertrifft sie in bezug auf Haltbarkeit. Die Erprobung unter Routinebedingungen zeigt, daß das Verfahren gut für das Kliniklaboratorium geeignet ist und die Ergebnisse mit denen der Glucoseoxydase/Peroxydase- und der Hexokinase-Methode übereinstimmen.

In the determination of blood sugar with glucose oxidase, the hitherto used peroxidase can be replaced by a mixture of iodide and vanadate. This inorganic catalyst is equal to peroxidase in colour yield, tate of reaction and susceptibility to interference, and it is more stable. Tests under routine conditions show that the method is well suited to the clinical laboratory, and the results agree with those from the glucose oxidase/peroxidase and the hexokinase methods.
\end{abstract}

Für die Routine-Blutzuckerbestimmung werden heute vor allem zwei Verfahren empfohlen: Die o-ToluidinMethode und das Glucoseoxydase ${ }^{\mathrm{I}}$-Verfahren.

Die o-Toluidin-Methode zeichnet sich durch eine erstaunlich hohe Spezifität aus (1-7). Es werden praktisch nur Aldohexosen erfaßt, so daß man nur bei dem seltenen Krankheitsbild der Galaktosämie und nach Belastung mit Galaktose mit Störungen rechnen muß

Die im zweiten Verfahren angewendete Glucoseoxydase (GOD) wird gern als Musterbeispiel für eine hohe Spezifität angegeben, da sie außer Glucose nur sehr wenige andere Verbindungen - und diese mit wesentlich geringerer Geschwindigkeit - oxydiert. Es bildet sich bei dieser Reaktion $\mathrm{H}_{2} \mathrm{O}_{2}$, das sich leicht durch Dianisidin oder Tolidin in Gegenwart von Peroxydase (POD) nachweisen läßt:

$$
\begin{aligned}
\text { Glucose }+\mathrm{O}_{2}+\mathrm{H}_{2} \mathrm{O} & \stackrel{[\text { GOD }]}{\longrightarrow} \text { Gluconsäure }+\mathrm{H}_{2} \mathrm{O}_{2} \\
\mathrm{H}_{2} \mathrm{O}_{2}+\text { Dianisidin } & \stackrel{[\text { POD }]}{\longrightarrow} \text { Farbstoff }
\end{aligned}
$$

1) Der Trivialname Glucoseoxydase wird hier gebraucht für das Enzym $\beta$-D-Glucose : $\mathrm{O}_{2}$-Oxydoreduktase EC 1.1.3.4; Peroxydase für Donor : $\mathrm{H}_{2} \mathrm{O}_{2}$-Oxydoreduktase EC 1.11.1.7; Hexokinase für ATP : D-Hexose-6-phosphotransferase EC 2.7.1.1.
Wegen der Spezifität der Glucoseoxydase-Reaktion hat man diese Bestimmung häufig als sehr genau angesehen: In manchen Fällen zu Unrecht, denn die Indikatorreaktion mit Peroxydase als solche ist unspezifisch. In Gegenwart von oxydierenden Substanzen, die schon durch geringe Verunreinigungen in die Probe gelangen können, können die Werte zu hoch ausfallen (8-9). Außerdem überträgt die Peroxydase das $\mathrm{H}_{2} \mathrm{O}_{2}$ auch auf viele andere Verbindungen (10) (z. B. auf Ascorbinsäure und Harnsäure), wodurch etwas zu tiefe Werte vorgetäuscht werden. Ferner enthalten handelsübliche Glucoseoxydase-Präparate häufig noch eine geringe Maltase-, Amylase- und Saccharase-Aktivität (11-15). Durch diese Fehlermöglichkeiten kann es trotz der hohen Spezifität der reinen Glucoseoxydase zu Abweichungen von den richtigen Glucose-Werten kommen.

Für Routinebestimmungen und für viele andere $Z$ wecke ist die Genauigkeit der Glucoseoxydase-Methode jedoch völlig ausreichend. Das Verfahren besitzt den Vorteil, $\mathrm{da} \beta$ es bei Zimmertemperatur durchgeführt werden kann. Schwierigkeiten treten im Vergleich zur o-Toluidin-Methode vor allem in bezug auf die Haltbarkeit auf: 
Die festen Reagenzien müssen im Kühlschrank aufbewahrt werden und die Puffer-Enzymlösung ist im Kühlschrank nur drei Wochen haltbar.

Die Funktionsfähigkeit der Puffer-Enzym-Lösung ist häufig durch die Qualität und die Haltbarkeit der Peroxydase begrenzt. Eine teilweise zersetzte Peroxydase liefert Eichkurven, die eine geringere Steigung besitzen und die nicht mehr linear verlaufen (Abb. 1). Nach der üblichen Berechnung erhält man dann im pathologisch erhöhten Bereich zu niedrige Werte. Deshalb ist es wünschenswert, die Peroxydase durch beständigere Verbindungen zu ersetzen.

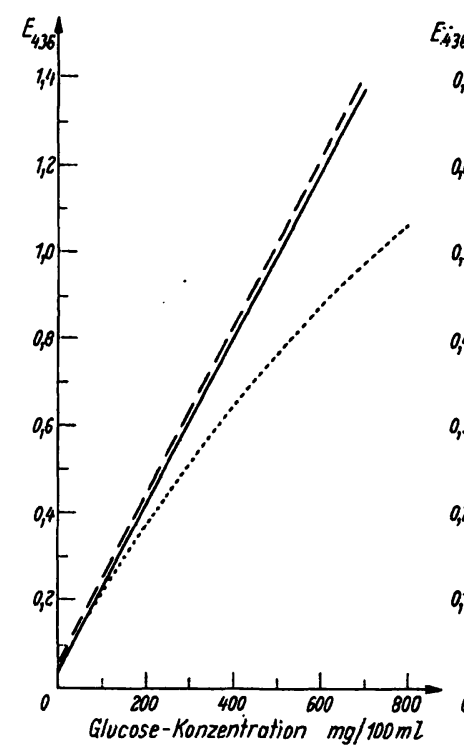

Abb. 1

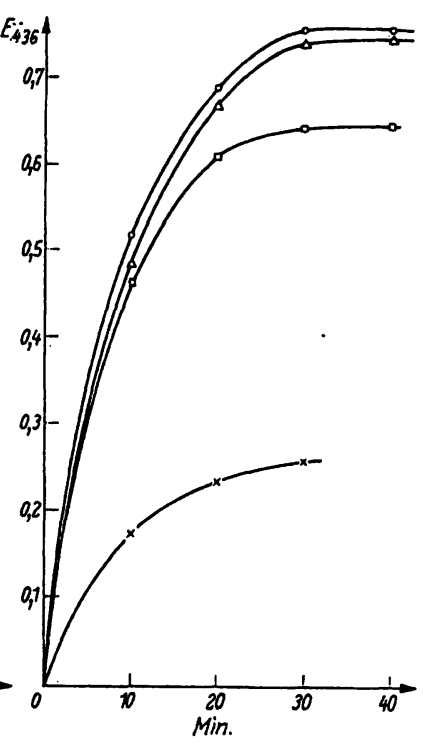

Abb. 2
Abb. 1

Eichkurven für die Blutzuckerbestimmung bei Verwendung von:

-...-..- Peroxydase

........ teilweise zersetzter Peroxydase

$\mathrm{KJ} / \mathrm{NH}_{4} \mathrm{VO}_{3}$

(0,2 ml Glucose-Lösung (1:11 verdünnt) $+5 \mathrm{ml}$ GOD-Reagenz, gegen Wasser gemessen)

Abb. 2

Farbentwicklung in Abhängigkeit von der Zeit bei Verwendung von Peroxydase und einiger anorganischer Redoxkatalysatoren $(0,2 \mathrm{ml}$ Glucose-Lösung $(80 \mathrm{mg} / 100 \mathrm{ml})+5 \mathrm{ml}$ GOD-Reagenz)

$\circ-\circ 4 \mathrm{mg}$ Peroxydase $/ 100 \mathrm{ml}$

$\triangle-\triangle 40 \mathrm{mg} \mathrm{NH}, \mathrm{VO}_{3}$ u. $400 \mathrm{mg} \mathrm{KJ} / 100 \mathrm{ml}$

- $2 \mathrm{~g}$ ( $\left.\mathrm{NH}_{4}\right)_{6} \mathrm{MO}_{3} \mathrm{O}_{24} \cdot 4 \mathrm{H}_{2} \mathrm{O}$ u. $2 \mathrm{~g} \mathrm{KJ} / 100 \mathrm{ml}$ (optimal)

$\times-x \quad 0,15 \mathrm{~g}\left(\mathrm{NH}_{4}\right)_{6} \mathrm{Mo}_{2} \mathrm{O}_{24} \cdot 4 \mathrm{H}_{2} \mathrm{O}$ u. $0,075 \mathrm{~g} \mathrm{KJ} / 100 \mathrm{ml}$ (17)

In der Literatur (16-19) sind folgende Verbindungen beschrieben, die zum Ersatz der Peroxydase (bzw. Peroxydase und Chromogen) dienen können: Kupfer-, Nickel-, Mangan- und Eisen-III-Salze der Äthylendiamintetraessigsäure; Eisen-II-Salze zusammen mit Gerbsäure oder Gallussäure oder Pyrogallussäure; Bleisulfid, Bleijodid, Kaliumjodid (gegebenenfalls zusammen mit Stärke oder o-Phenylendiamin, Tolidin oder Wolframaten und Tolidin). Außerdem ist die Verwendung von Blut, Erythrocyten und von MetallsalzKomplexen mit Porphyrinen (z. B. Hämin) oder Phthalocyaninen zusammen mit Aminodonatoren (Aminobenzothiazol, Pyridin, Nicotinsäure und Bipyridylpyridin) möglich $(20,21)$. Ferner sind noch eine große $\mathrm{Zahl}$ anderer organischer Verbindungen ( $z$. B. verschiedene aliphatische Aldehyde, phosphororganische Verbindungen, Nucleinsäure usw.) beschrieben (10), die die Übertragung von $\mathrm{H}_{2} \mathrm{O}_{2}$ auf Chromogene katalysieren, jedoch noch nicht für die Blutzuckerbestimmung verwendet wurden.

Keine dieser vielen Verbindungen hat jedoch eine größere Anwendung gefunden, da die Farbausbeuten schlechter als bei Verwendung von Peroxydase sind. Für Routinezwecke ist das System
Molybdat/Jodid/Tolidin bedingt brauchbar. Jedoch ist bei der vorgeschlagenen Methode (18) das Tolidin im Reagenz'so wenig haltbar, daß es erst unmittelbar vor Ausführung der Analyse zugesetzt werden darf. Es ist dabei eine zusätzliche Pipettierung erforderlich. Außerdem sind die Blindwerte sehr hoch. Diese Nachteile kann man vermeiden, wenn man an Stelle von Acetatpuffer $(\mathrm{pH}=4,5)$ und Tolidin Phosphatpuffer $(\mathrm{pH}=7,0)$ und Dianisidin verwendet. Durch Vergrößerung der Katalysatorkonzentration kann die Farbausbeute von 30 auf $80 \%$ (bezogen auf die Verwendung von Peroxydase) gesteigert werden.

Wesentlich besser ist jedoch das Katalysator-System Jodid/Vanadat für die Blutzuckerbestimmung geeignet. Im Gegensatz zum Molybdat, das bei einer optimalen Katalysatorkonzentration von $1-2 \%$ immer noch im Vergleich zur Peroxydase unbefriedigende Farbausbeuten liefert, wirkt Vanadat schon in Konzentrationen von $0,04 \%$. Selbst in dieser geringen Konzentration erhält man Farbausbeuten wie bei Verwendung von Peroxydase. Darüber hinaus besitzt die Verwendung dieses Katalysators an Stelle von Peroxydase den Vorzug, daß er in reiner Form zur Verfügung steht und unter den üblichen Bedingungen keine Haltbarkeitsprobleme auftreten.

\section{Methodik}

Für die Blutzuckerbestimmung werden die Reagenzien aus der Testpackung Merckotest Blutzucker (GOD-Methode) verwendet. Die Durchführung erfolgt nach folgendem Schema:

Löșungen

Enteiweißungsgemiscls: $0,0038 \mathrm{M}$ Uranylacetat $0,16 \mathrm{M} \quad \mathrm{NaCl}$

Puffer-Enzymgemiscl: : 0,12M Phosphatpuffer

$0,02 \mathrm{M}$ Trishydroxymethylaminomethan $0,25 \mathrm{mg} \mathrm{GOD} / \mathrm{ml}(25 \mathrm{U} / \mathrm{mg})$

$0,024 \mathrm{M} K \mathrm{~J}$

$0,0034 \mathrm{M} \mathrm{NH}_{4} \mathrm{VO}_{3}$ oder

$0,0014 \mathrm{M} \mathrm{Na}_{3} \mathrm{VO}_{4}$

Dianisidin-Lösıng: $\quad 0,023 \mathrm{M}$ Dianisidin

Farbreagenz (nur den Tagesbedarf ansetzen!): $50 \mathrm{~m} l$ Puffer-Enzymgemisch und $0,5 \mathrm{~m} l$ Dianisidin-Lösung vermischen.

Standardlösung: $100 \mathrm{mg}$ Glucose (wasserfrei) $/ 100 \mathrm{ml}$.

Ausführung

$\mathrm{Zu}$ jeder Analyse werden nur ein bis zwei Standardproben angesetzt Enteiweißung: In Zentrifugengläser einpipettieren:

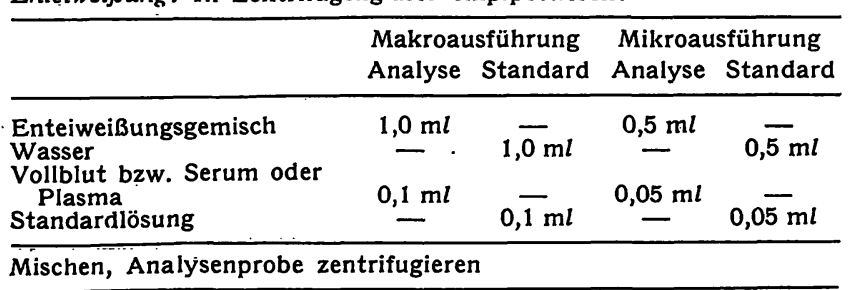

Farbreaktion: In Reagenzgläser einpipettieren :

Makroausführung Mikroausführung

Analyse Standard Analyse Standard

\begin{tabular}{lllll}
\hline $\begin{array}{l}\text { Eiweißfreie Analysen-Lösung } \\
\text { bzw. Standardgemisch }\end{array}$ & $0,2 \mathrm{ml}$ & $0,2 \mathrm{ml}$ & $0,1 \mathrm{ml}$ & $0,1 \mathrm{ml}$ \\
Farbreagenz & $5,0 \mathrm{ml}$ & $\mathbf{5 , 0} \mathrm{ml}$ & $\mathbf{3 , 0 \mathrm { ml }}$ & $\mathbf{3}, 0 \mathrm{ml}$ \\
\hline
\end{tabular}

Mischen, bei Zimmertemperatur stehen lassen. Nach 30 Min. die Extinktionen der Analysen und der Standardproben gegen das Farbreagenz messen

Filter: zwischen $420-470$ nm, z. B. $\mathrm{Hg} 436$

Schichtdicke: $1 \mathrm{~cm}$ 
Tab. 1

Einfluß einiger Serumzusätze auf die Vanadat- und auf die POD-Methode

Um auch extrem hohe Konzentrationen prüfen zu können, wurden die Zusätze nicht ins Serum, sondern in den eiweißfreien Uberstand gegeben. Die Werte wurden anschließend auf Serum umgerechnet.

\begin{tabular}{|c|c|c|c|c|}
\hline Zusatz & $\begin{array}{c}\text { Konzentration in } \\
\mathbf{3} \% \text { Störun } \\
\text { Vanadat-Methode } \\
\mathrm{mg} / \mathrm{ml}\end{array}$ & $\begin{array}{l}\text { Serum, die etwa } \\
\text { verursacht } \\
\text { POD-Methode } \\
\text { mg/ml }\end{array}$ & Bemerkung & $\mathrm{mg} / \mathrm{ml}$ \\
\hline $\begin{array}{l}\text { NaF } \\
\text { EDTA, Dinatriumsalz } \\
\text { Na-Citrat } \\
\text { Na-Oxalat } \\
\text { Ascorbinsäure } \\
\text { Glutathion }\end{array}$ & $\begin{array}{r}>1000 \\
>\quad 100 \\
>\quad 100 \\
30 \\
0,02 \\
4\end{array}$ & $\begin{array}{r}>1000 \\
>\quad 100 \\
100 \\
30 \\
0,03 \\
4\end{array}$ & \multirow{2}{*}{$\begin{array}{l}\text { Zur Gerinnungshemmung: } \\
\text { Zur Gerinnungshemmung: } \\
\text { Zur Gerinnungshemmung: } \\
\text { Zur Gerinnungshemmung: } \\
\text { Normalwert: } \\
\text { Normalwert Serum: } \\
\text { Normalwert Vollblut: } \\
\text { Zur Verhinderung des Wachstums von Mikro- } \\
\quad \text { organismen: }\end{array}$} & \multirow{2}{*}{$\begin{array}{r}3 \\
1 \\
10 \\
2 \\
0,01 \\
0,00 \\
0,02 \\
1\end{array}$} \\
\hline $\mathrm{NaN}_{3}$ & 10 & 10 & & \\
\hline
\end{tabular}

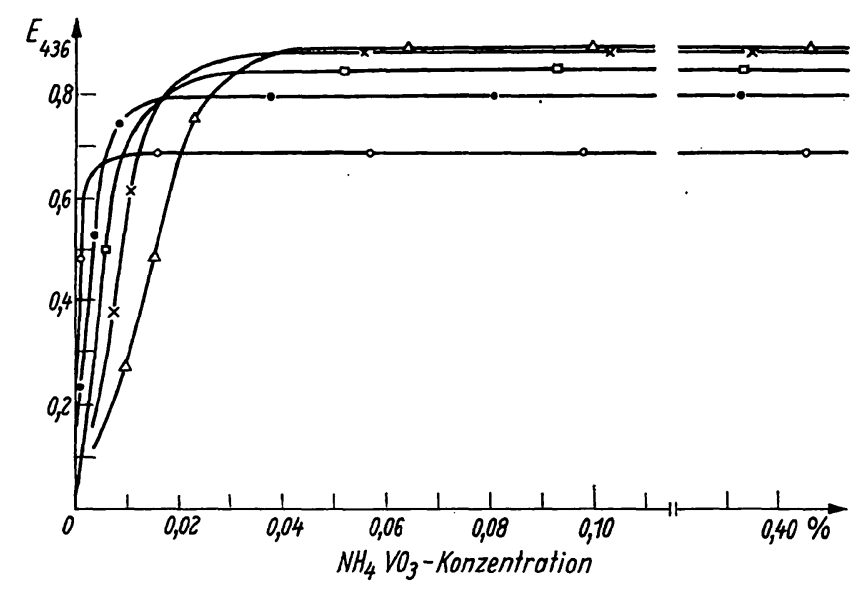

Abb. 3

Abhängigkeit der Farbausbeute von der $\mathrm{KJ}$ - und der $\mathrm{NH}_{4} \mathrm{VO}_{3}$ Konzentration.

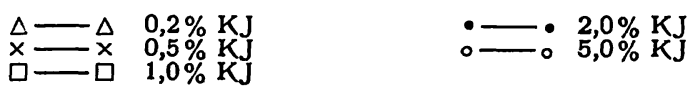

\section{Ergebnisse und Diskussion}

Optimale Bedingungen

Die Ergebnisse der Versuche zur Ermittlung optimaler Reaktions-Bedingungen sind in den Abbildungen 2-4 und in der Tabelle 1 dargestellt. Aus den angegebenen Vergleichsdaten ist ersichtlich, daß in bezug auf Farbausbeute, Reaktionsgeschwindigkeit und Störanfälligkeit kaum Unterschiede zwischen der Peroxydase- und der Vanadat-Methode bestehen.

Wie bei Verwendung von Peroxydase können die Nebenaktivitäten der Glucoseoxydase zum größten Teil durch einen Zusatz von Trispuffer gehemmt werden. Von den untersuchten Chromogenen ist o-Dianisidin am besten geeignet. Außerdem können noch oTolidin, Benzidin, p-Toluidin, o-Naphthol, p-Dianisidin, Diaminofluoren und o-Phenylendiamin verwendet werden. Weniger gut geeignet sind N,N-Dimethylphenyldiamin, Guajakol und Pyrogallol. Dianisidin verwendet man am besten in Form des Bisdihydrogenphosphats. Die Chromogenlösung besitzt dann einen etwas geringeren $\mathrm{pH}$ als bei Verwendung des Hydrochlorids und ist besser haltbar.

Zur Enteiweißung wird Uranylacetat bevorzugt, da sich hierbei ein sehr gleichmäßiger, feinkörniger Niederschlag bildet und da sich Eiweiß und Enteiweißungsmittel gegenseitig quantitativ ausfällen. Wenn man eine eiweißhaltige Standardprobe verwendet, wird diese wie die Analysenproben enteiweißt. Einfacher ist jedoch die Verwendung einer Lösung einer bekannten Menge Glucose (Wassergehalt der käuflichen Präparate berücksichtigen!) in Wasser. Man muß jedoch darauf achten, daß hierbei an Stelle des Enteiweißungsschrittes mit Wasser und nicht mit Uranylacetatlösung verdünnt wird (wie bei Verwendung von Peroxydase). Sonst ent-

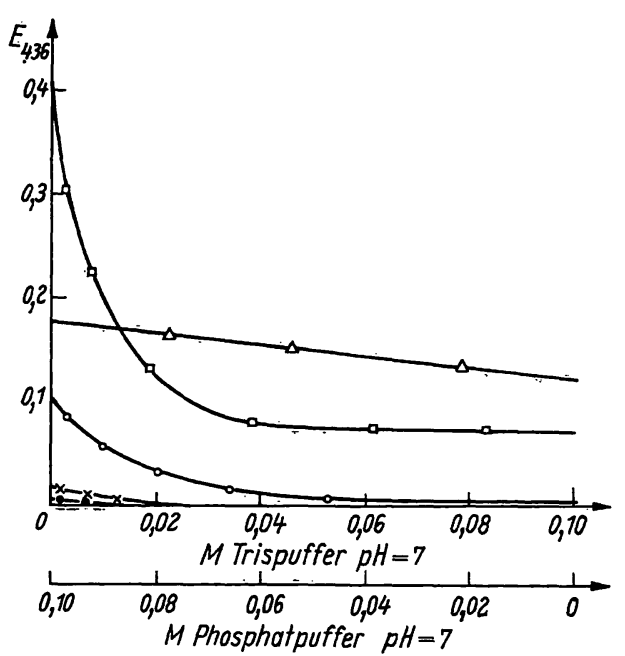

Abb. 4

Hemmung von Nebenaktivitäten der Glucoseoxydase durch Trishydroxymethylaminomethan.

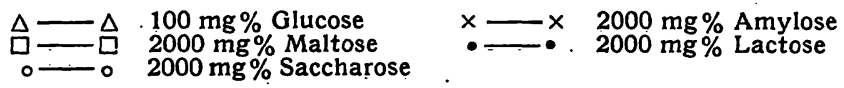

hält die Standardlösung Uranylacetat, wodurch sich etwas zu hohe Standardextinktionen ergeben.

Man kann jedoch die Analysenproben auch sauer enteiweißen, was insbesondere wegen der oben erwähnten Fehlermöglichkeit immer dann erforderlich ist, wenn die Proben sehr stark im Eiweißgehalt von dem des Serums bzw. Blutes abweichen (z. B. Liquor). Im allgemeinen sollte hierbei Trichloressigsäure bevorzugt werden, $\mathrm{da}$ sie nach dem Zentrifugieren klare Uberstände liefert. Nur wenn aus demselben Uberstand noch Parallelbestimmungen mit Hilfe der Hexokinase-Methode durchgeführt werden sollen, verwendet man Perchlorsäure, da das Hexokinase-Verfahren durch Trichloressigsäure gestört wird. Bei der sauren Enteiweißung sollte man in jedem Fall das unterschiedliche Verhalten der o- und der mVanadate beachten. Während bei den stabileren o-Vanadaten keine Schwierigkeiten auftreten, bilden die m-Vanadate in stark saurer Lösung leicht gelbe Polyvanadate. Deshalb enteiweißt man bei Verwendung von $\mathrm{m}$-Vanadaten mit einer partiell neutralisierten Säure (z. B. mit einer Lösung von $3 \mathrm{~g}$ Trichloressigsäure in 1 proz. Natronlauge). Hierdurch kann man beim Zugeben des sauren Uberstandes zum Farbreagenz ganz sicher einen örtlich zu geringen $\mathrm{pH}$ und dadurch etwas höhere Blindwerte vermeiden.

Wegen der Empfindlichkeit der Methode gegenüber Verunreinigungen empfiehlt es sich, die Linearität der Eichkurve zu überprüfen, wenn man die einzelnen Substanzen für die Bereitung des Reagenzes selbst zusammenstellt. Hierbei muß man besonders auf den Bereich sehr kleiner Glucosekonzentrationen achten. Wenn hier in der Eichkurve eine Krümmung auftritt, erhält man nach der üblichen Berechnung bei Proben mit seht hohem Blutzuckergehalt leicht $\mathrm{zu}$ hohe Werte. Im Routinebetrieb erkennt man diesen Fehler häufig an zu geringen Standardwerten. Bei Verwendung ausreichend reiner Reagenzien kommt dieser Fehler jedoch nicht vor. 


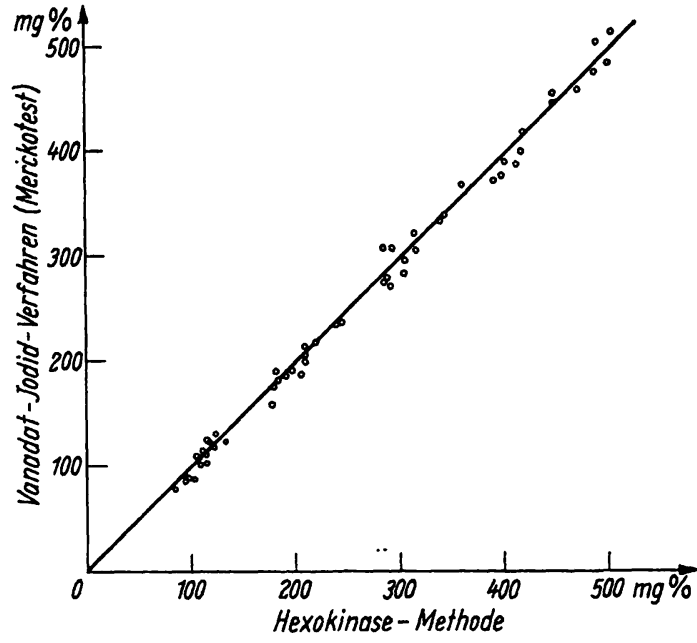

Abb. 5

Vergleich der GOD-Vanadat-Jodid-Methode mit der HexokinaseMethode

Vergleich der verschiedenen enzymatischen Methoden zur Blutzuckerbestimmung

Zur Erprobung der Brauchbarkeit der neuen Methode wurden eine größere Zahl Proben nach dem GODVanadat-Jodid,- nach dem GOD-POD- und nach dem Hexokinase-Verfahren untersucht. (Beim HexokinaseVerfahren wurde hierbei nicht wie üblich mit der Enzymsuspension, sondern mit dem eiweißfreien Überstand gestartet, da sonst bei der Untersuchung von Standardlösungen nicht immer die theoretischen Werte erhalten wurden.) Es handelte sich um Blutproben von Blutspendern bei Glucosebelastungsversuchen, um Seren aus einem Kliniklaboratorium und wegen der Störanfälligkeit der Methode bei hohen Glucosegehalten um

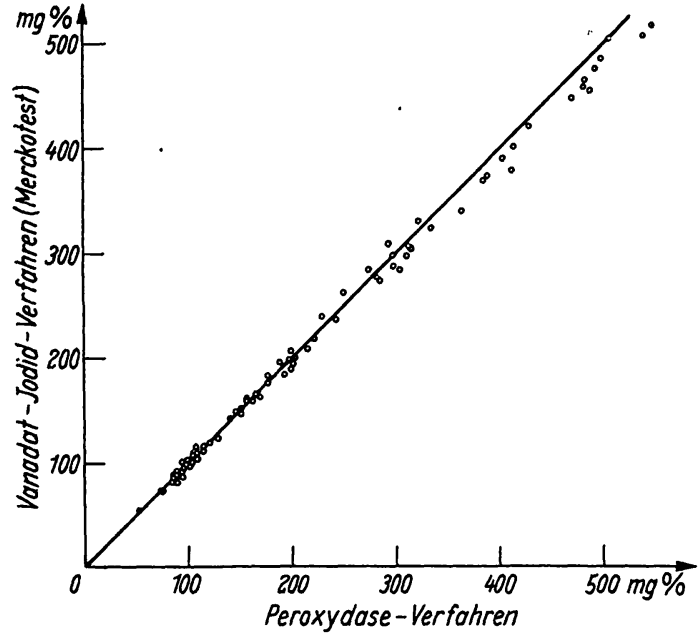

Abb. 6

Vergleich der GOD-Vanadat-Jodid-Methode mit der GOD-PODMethode

Seren mit einem Glucosezusatz. Aus den in den $\mathrm{Ab}$ bildungen 5 und 6 dargestellten Ergebnissen (Einzelbestimmungen, 1 Standard pro Serie) ersieht man, da $B$ die Werte hinreichend gut mit denen der Vergleichsmethoden übereinstimmen. Die Vergleichsstandardabweichung (gemessen an denselben eiweißfreien Überständen in drei verschiedenen Laboratorien $\mathrm{zu}$ verschiedenen Tageszeiten) beträgt an der oberen Grenze des Normalbereiches $\pm 3 \mathrm{mg} / 100 \mathrm{~m} l$ und ist damit genau so groß wie der entsprechende, bei Verwendung von Peroxydase ermittelte Wert.

Wir danken Fräulein R. Finkenauer, Frau A. Hrmsel und Fräulein H. GOECKE für die gewissenhafte Durchführung der Versuche.

\section{Literatur}

1. Dubowskx, K. M., Clin. Chem. (New York) 8, 215 (1962). - 2. ZeNDER, R., Clin. chim. Acta (Amsterdam) 8, 351 (1963). - 3. Watson, D. und M. E. K. Stevenson, Austral. J. exp. Biol. med. Sci. 41, 211 (1963). - 4. Ahlert, G., E. Hofer, W. HoffmanN und G. Bestvater, Dtsch. Gesd.wes. 19, 2256 (1964); 20, 349 (1965). - 5. HruärInen, A. und E. A. NrkxirA, Clin. chim. Acta (Amsterdam) 7, 140 (1962). - 6. MüLIER, G., Dtsch. Zschr. Verdauungs- und Stoffwechselkr. 25, 76 (1965). - 7. ZENDER, R. und S. NolneN, Med. Laborat. 19, 214 (1966). - 8. SCGHMIDT, F. H., in: Fortschritte der Diabetesforschung, S. 76. Georg Thieme Verlag, Stuttgart (1963). - 9. Fales, F. W. und D. SeLIGson, Stan. dard Methods Clin. Chem. 4, 101 (1965). - 10. Saunders, B. C.,
A. G. Stark und A. G. Holmes-Siedle, Peroxydase, S. 173-177 und 201-213. Butterworth \& Co., London (1964). - 11. SCHrweCK, H., Zucker 16, 170 (1963). - 12. WhISTLER, R. L., L. Hovgr und I. W. Hrun, Analytic. Chem. 25, 1215 (1953) - 13. Dahlquist, A., Biochem. J. 80, 547 (1961). - 14. TÄUfEL, K., H. RutrLoff und R. FrIESE, Naturwissenschaften 49, 375 (1962). - 15. Fleming, I. D. und H. F. Pegler, Analyst 88, 967 (1963). - 16. Can. P. 24,060. - 17. Bel. P. 627, 415. - 18. Trompson, R. H., Clin. chim. Acta (Amsterdam) 13, 133 (1966). - 19. Malmstedt, H. V., H. L. Pardue und S. I. Hadjiroannou, Analytic. Chem. 34, 542 (1962); 33, 1040 (1961). — 20. D. B. P. 1185841. - 21. D. B. P. 1121846.

Dr. A. Härtel

E. Merck A. G., Biochemische Abteilung 61 Darmstadt, Postfach 4119 